\title{
ECOLOGIE DES CRUSTACÉS BENTHIQUES DU LAC DE PORT-BIELH (PYRENEES CENTRALES) : II. CYCLES BIOLOGIQUES
}

\author{
par J. ReY ${ }^{1}$ et B. Dupin ${ }^{1}$.
}

\begin{abstract}
Les cycles biologiques de quelques populations de Cladocères et de Copépodes d'un lac de haute montagne ont été suivis de juin à novembre 1971, période où les eaux étaient dégagées des glaces. Dans la plupart des cas le cycle vital des espèces (croissance, reproduction) se déroule durant la période estivale, le lac étant gelé le reste de l'année. La majorité des espèces sont monocycliques. Le cycle des Cladocères débute au moment du dégel avec l'éclosion des œufs de durée, suivie d'un maximum de reproduction parthénogénétique en août - septembre. Une phase générale de gamogénèse marque la fin du cycle. Les Copépodes sont déjà en cours de reproduction au moment du dégel; le renouvellement des populations s'effectue pendant l'été. Les espèces s'adaptent aux conditions extrêmes du milieu en adoptant des processus morphologiques et physiologiques particuliers : éphippies de Cladocères, enkystement et phase de dormance des Copépodes.
\end{abstract}

\section{Ecology on the benthic Crustacea \\ in Lake Port-Bielh (central Pyrenees) : II. Biological cycles.}

The biological cycles of several populations of Cladocera and Copepoda of a high mountain lake have been followed from June to November 1971, a period when the lake is free of ice. In most cases, the life cycle of the species (growth, reproduction) was extended over the summer period, the lake being frozen for the rest of the year. Most species are monocyclic. The cycle of the Cladocera starts with the hatching of resting eggs when the ice melts. and then follows a period of maximum parthenogenetic reproduction in August and September. A general phase of gamogenesis marks the end of the cycle. The Copepoda are already reproducing when the ice melts; the renewal of populations occurs in summer. The species adapt to the extreme conditions of the environment by exhibiting special morphological and physiological characteristics : ephipia of Cladocera, encystment and a dormant phase of Copepoda.

\section{INTRODUCTION}

Parmi les nombreux travaux réalisés sur le benthos des lacs de montagne, la grande majorité concerne le macrobenthos (Chironomides, Oligochètes, Mollusques). Le microbenthos et les Crustacés

1. Laboratoire d'Hydrobiologie, Université Paul-Sabatier, 118, route de Narbonne, 31077 Toulouse Cedex. 
en particulier sont généralement délaissés. Ce manque de données sur les Crustacés benthiques s'explique par les difficultés d'estimation quantitative de ces populations, difficultés dues à la fois à l'imperfection des méthodes actuelles de prélèvement, à la distribution irrégulière de la faune et à l'hétérogénéité du substrat. Cependant, du fait du développement d'importantes populations, les Crustacés représentent parmi les communautés benthiques un ensemble non négligeable.

Dans une première note (Rey et Dupin 1973), nous avons considéré la structure et l'évolution du peuplement en Cladocères, Copépodes et Ostracodes du lac de Port-Bielh, lac d'origine glaciaire situé à $2285 \mathrm{~m}$. Cette étude a montré que l'essentiel de la biomasse était constitué par quelques espèces dominantes, le facteur thermique jouant un rôle primordial dans l'évolution des populations. Les observations réalisées en 1970 et 1971 dans le lac de Port-Bielh nous ont permis de préciser les cycles évolutifs des principales espèces, cycles dont l'étude fait l'objet du présent travail.

\section{I. - MATÉRIEL ET METTHODES}

Les principales caractéristiques morphométriques et physicochimiques du milieu ayant été exposées dans la publication précédente (Rey et Dupin 1973), nous renvoyons à cette dernière, ou, pour des données plus détaillées, au travail de Capblancq et Laville (1968).

Pour chaque espèce, les cycles évolutifs ont été établis à partir de prélèvements effectués au cours de l'été 1971, environ tous les dix jours, du 22 juin (début du dégel du lac) au 5 novembre. En outre, des informations complémentaires ont été apportées par l'examen de récoltes de l'année précédente et de la période hivernale. Ces prélèvements ont été réalisés toujours à la même profondeur : - $1 \mathrm{~m}$ dans la zone "littorale», $-13 \mathrm{~m}$ dans la zone "littori-profonde». Toutefois, l'évolution de Cyclops strenuus, espèce pseudo-benthique, a été suivic à la fois à partir de prélèvements benthiques et à partir d'individus récoltés dans les flacons de pièges à émergence de Chironomides.

Seuls ont été considérés les cycles évolutifs des espèces de Cladocères et Copépodes toujours représentées dans les prélèvements par un nombre suffisant d'individus. Afin d'obtenir des cycles évolutifs les plus précis possible, pour chaque espèce étudiée, les catégories suivantes d'individus ont été décomptées :

- Cladocères :

* jeunes (catégorie groupant tous les stades jeunes : néonatae, formes juvéniles, stades immatures); 
* q ovigères;

* q non ovigères ;

* $q$ éphippiales ;

* $\delta$.

- Copépodes Cyclopides et Harpacticides :

* stades copépodites 1 à 5 ô et $\%$;

* $q$ ovigères ;

* q non ovigères;

* $\delta$.

Il n'a pu être tenu compte des nauplii, une grande partie d'entre eux ayant échappé à nos observations soit en raison de la difficulté à les séparer des particules de vase, soit à cause du vide de maille trop important pour certains stades naupliens.

\section{II. - RESULTATS}

Pour les Cladocères, les résultats sont exprimés en nombre d'individus par $\mathrm{m}^{2}$, et représentés par des courbes figurant l'évolution dans le temps des différentes catégories d'individus définis ci-dessus. Toutefois, dans un but de simplification, seules ont été représentées les populations de jeunes, $q$ ovigères et $q$ éphippiales, catégories les plus significatives pour l'établissement du cycle annuel.

En ce qui concerne les Cyclopides, le type de prélèvement utilisé pour Cyclops strenuus (récolte dans les pièges à émergence) ne permettant pas de donner des valeurs absolues, le nombre des individus de chaque catégoric a été représenté en pourcentage par rapport au nombre total des individus de l'espèce considérée. Dans un souci de comparaison, ce mode d'expression a été adopté pour tous les Cyclopides.

\section{Cladocères (fig, 1).}

Les observations ont porté sur deux populations littorales : Alona affinis et $A$. quadrangularis et quatre populations de la zone "littori-profonde»: Eurycercus lamellatus, Acroperus harpae, Simocephalus vetulus, llyocryptus sordidus.

Pour toutes ces espèces, le cycle évolutif répond au même schéma : les espèces se développent plus ou moins tôt dans la saison selon les conditions de milieu (dégel plus ou moins précoce, régime thermique). L'éclosion des aufs de durée se déclenche dès 

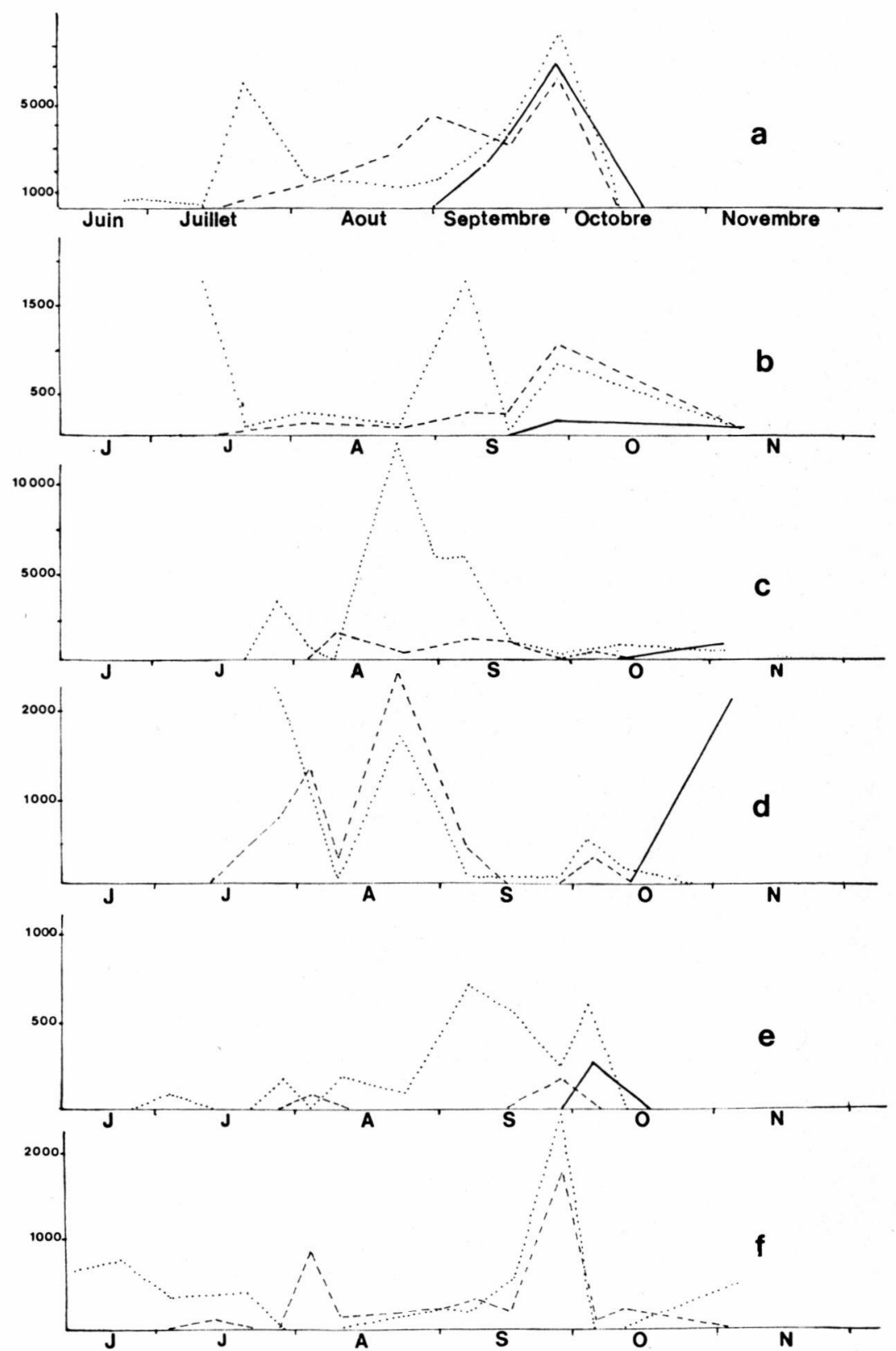

Fig. 1. - Evolution de six populations de Cladocères benthiques, au cours de l'année 1971, dans le lac de Port-Bielh :

$1^{\circ}$ Au niveau de la zone «littorale»: a. Alona affinis; b. Alona quadrangularis;

$2^{\circ}$ Au niveau de la zone «littori-profonde»: c. Eurycercus lamellatus; d. Acroperus harpae; e. Simocephalus vetulus; f. Ilyocryptus sordidus.

$\ldots \ldots$ : jeunes;

- . . . : : o ovigères;

- 
le réchauffement des eaux. Par suite d'une active reproduction parthénogénétique, les populations atteignent une densité maximale en août-septembre. Ces maxima sont toujours suivis d'une chute brutale du nombre des individus annonçant le déclin de la population. La plupart des espèces sont monocycliques. On observe une seule phase de gamogénèse, automnale, plus ou moins avancée selon les espèces, mais qui sanctionne netlement la fin du cycle vital avec formation d'œufs de durée. Une évolution semblable avait été observée (Rey 1968) chez des populations de Cladocères peuplant des lacs voisins sensiblement à la même altitude. Deux espèces toutefois font exception à ce schéma :

* Ilyocryptus sordidus dont la population suit cependan! une évolution comparable à celle des autres espèces avec une nette phase de déclin en novembre, n'a montré ni $\hat{o}$ ni $q$ éphippiales. Ainsi pour cette espèce, phase de gamogénèse ou formes de résistance soit ont échappé à nos observations, soit se sont produites plus tardivement en période hivernale;

* Acroperus harpae, par contre, semble avoir un comportement différent. En effet, l'examen d'un prélèvement d'hiver effectué le 22 avril sous la glace à $16 \mathrm{~m}$ de profondeur, a révélé de nombreux individus en pleine phase de reproduction parthénogénétique, alors qu'en novembre la population était représentée uniquement par sa forme de résistance. Contrairement aux autres espèces il semble qu'Acroperus harpae soit dicyclique dans le lac de Port-Bielh.

\section{Cyclopides (fig. 2).}

L'étude du cycle évolutif de Paracyclops fimbriatus, Eucyclops serrulatus, Cyclops strenuus, Macrocyclops fuscus révèle pour chacune de ces espèces une évolution identique des populations :

- au moment du dégel, les adultes sont en phase de reproduction. Le nombre de $\%$, ovigères ou non ovigères, est de beaucoup supérieur à celui des $\delta$;

- de la mi-août à la mi-septembre, la population présente un maximum de stades copépodites;

- en fin de saison estivale est apparue une nouvelle généralion. Le peuplement est alors essentiellement constitué par des adultes ( $\hat{f}, q$ non ovigères) et des stades copépodites avancés (copépodites IV et $\mathrm{V}$ ).

Toutes ces espèces paraissent monocycliques, le renouvellement de la population s'effectuant pendant l'été. Seul Eucyclops serrulatus présente fin septembre un début de reproduction. Ces espèces arrivent à l'entrée de l'hiver à l'état d'adultes ou de stades subadultes et semblent, par suite de l'apparition de conditions défavo- 


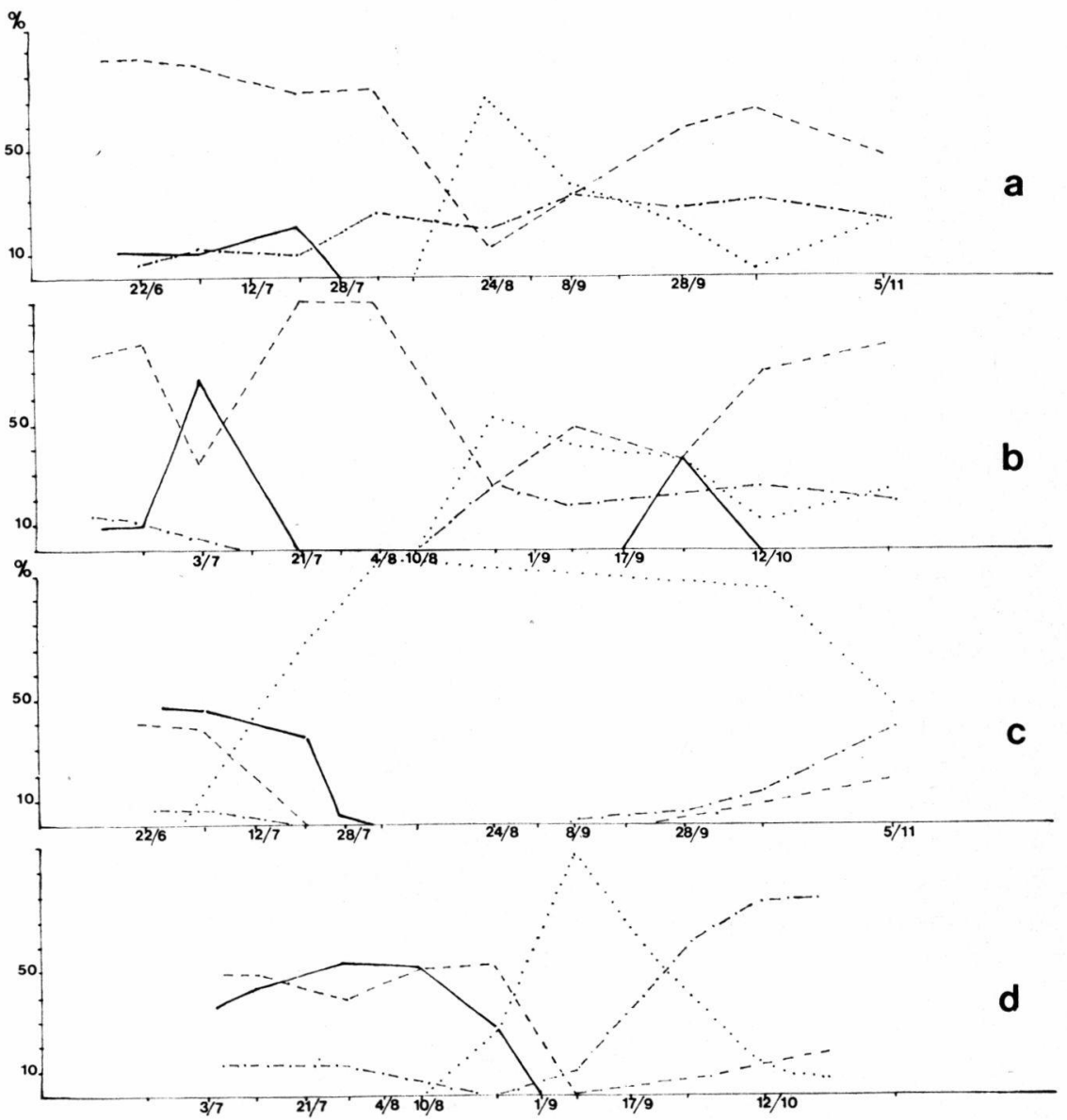

Fig. 2. - Evolution de quatre populations de Cyclopides, au cours de l'année 1971, dans le lac de Port-Bielh : a. Paracyclops fimbriatus; b. Eucyclops serrulatus; c. Cyclops strenuus; d. Macrocyclops fuscus. ....... : stades copépodites;

-.-. - : : 8 ;

- - . - : : non ovigères;

: o ovigères.

rables (baisse des températures et du taux d'oxygène), passer l'hiver sous ces différentes formes.

\section{Harpacticides.}

Le cycle biologique de Canthocamptus staphylinus (fig. 3) se raccorde au schéma général du cycle évolutif des Cyclopides. Toutefois il offre une plus grande complexité par le fait que cette 


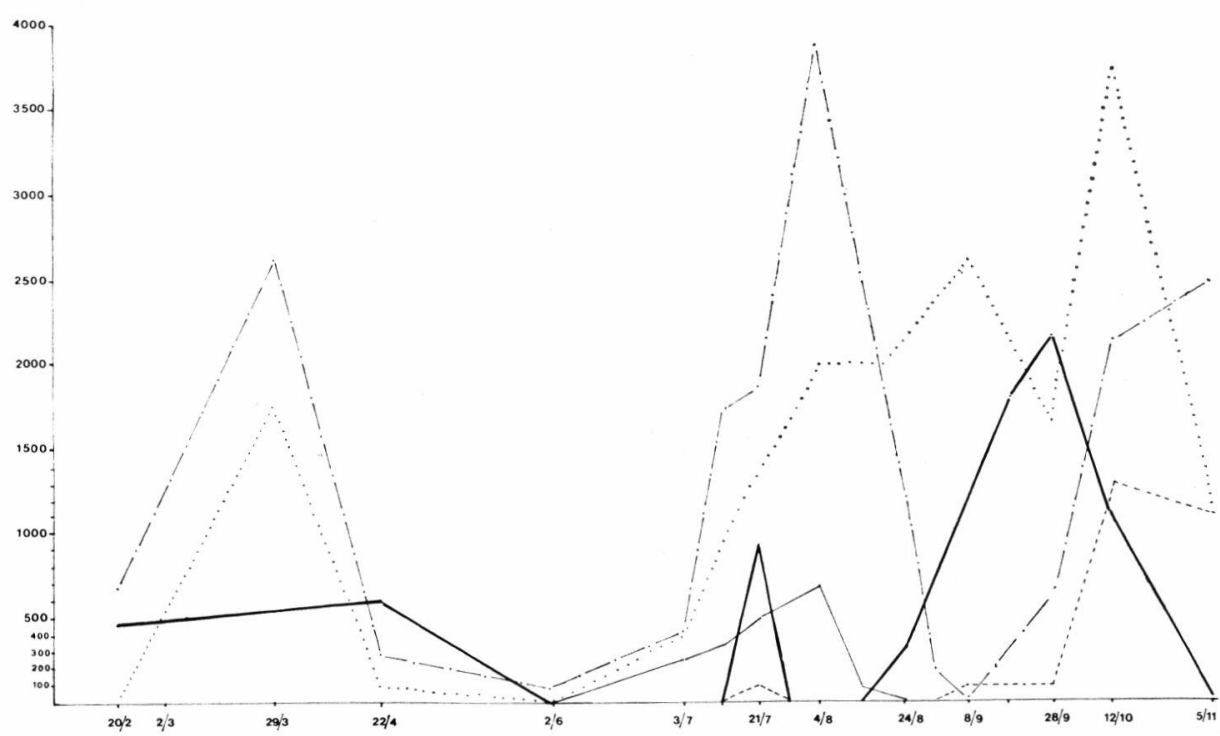

Fig. 3. - Evolution de la population de Canthocamptus staphylinus, dans le lac de Port-Bielh, au cours de l'année 1971, en nombre d'individus par m². -.... : $\hat{\delta}$;

$\ldots \ldots . .$. : $q$ non ovigères ;

-. - - - : ᄋ à spermatophores;

-. - - - : 9 ovigères;

- : kystes.

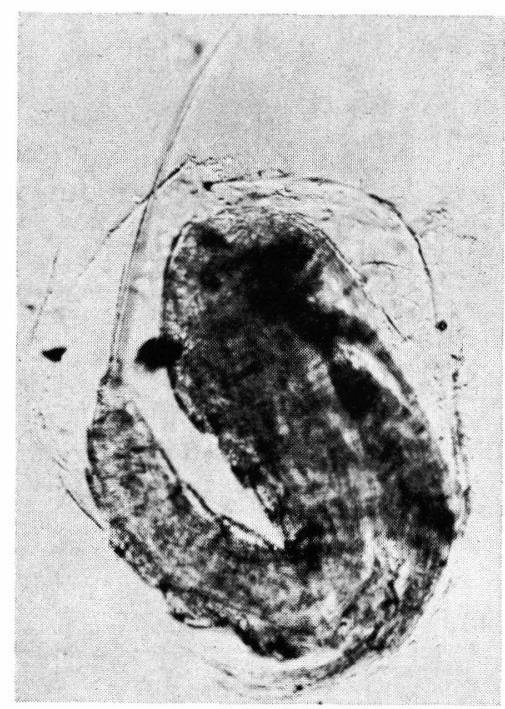

FIG. 4. - Canthocamptus staphylinus : individu enkysté. 
espèce présente un enkystement tout au long de son cycle vital.

Les kystes récoltés dans le lac de Port-Bielh rappellent les observations de K. Berg (1938) : l'animal est entouré d'une membrane hyaline et replié sur lui-même par flexion dorsale du postabdomen (fig. 4). Des kystes semblables avaient déjà été observés dans un lac voisin (Gouxette 1966). Ces phénomènes d'enkystement se produisent tant en été qu'en hiver (fig. 5).

L'enkystement estival semble caractérisé par une discontinuité dans l'apparition des kystes, de telle sorte que l'on peut distinguer des kystes de début été et des kystes de plein été.

Les kystes de début été apparaissent un certain laps de temps (environ 3 semaines) après le dégel : en 1970, ils ont été observés le 6 août (dégel vers la mi-juillet); en 1971, ils ont été observés le 21 juillet (dégel : fin juin - début juillet). Ces kystes sont relativement éphémères; on ne les observe guère que durant une période de dix jours.

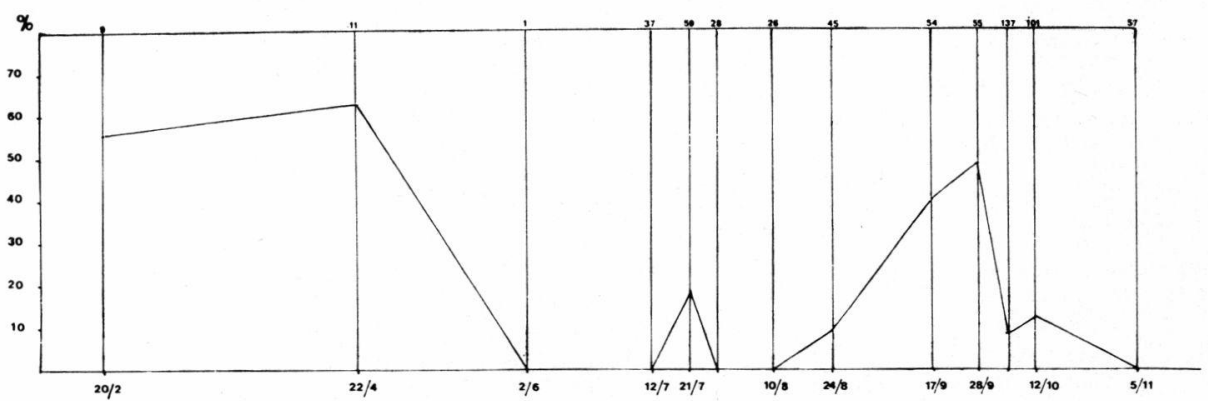

Fig. 5. - Rapport kystes - individus de Canthocamptus staphylinus en période hivernale et en période estivale.

Les kystes de plein été ont été rencontrés à partir des mêmes dates en 1970 et 1971 : observés dès le 4 août, ils disparaissent entre le 12 octobre et le 5 novembre l'une et l'autre année. Leur production maximale se situe en septembre où ils représentent presque $50 \%$ de la population totale. Le phénomène touche surtout les $q$ à spermatophores. Ainsi sur 76 kystes d'été, les proportions d'individus enkystés sont les suivantes :

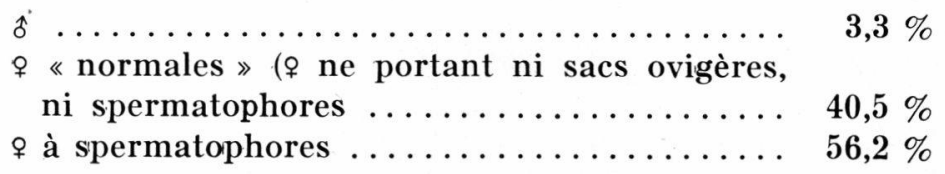

Enkystement hivernal : les kystes hivernaux provenant de prélèvements effectués sous la glace à $13 \mathrm{~m}$ de profondeur, ont été 
observés dès février, jusqu'à fin avril. Sur 20 individus récoltés, 12 étaient enkystés parmi lesquels 2 ô et 10 ㅇ $(8 \%$ «normales » et 2 ㅇ à spermatophores). A côté de ces individus nettement enkystés quelques $q$ «normales» ou à spermatophores ne présentaient pas de membrane kystique mais montraient cependant (fig. 6) une flexion dorsale du post-abdomen, plus ou moins accentuée. Audelà d'avril et jusqu'au mois de juin, il a été trouvé de nombreux kystes vidés de leurs individus (préparation à la reprise d'une vie active et à la phase de reproduction de juillet ?).
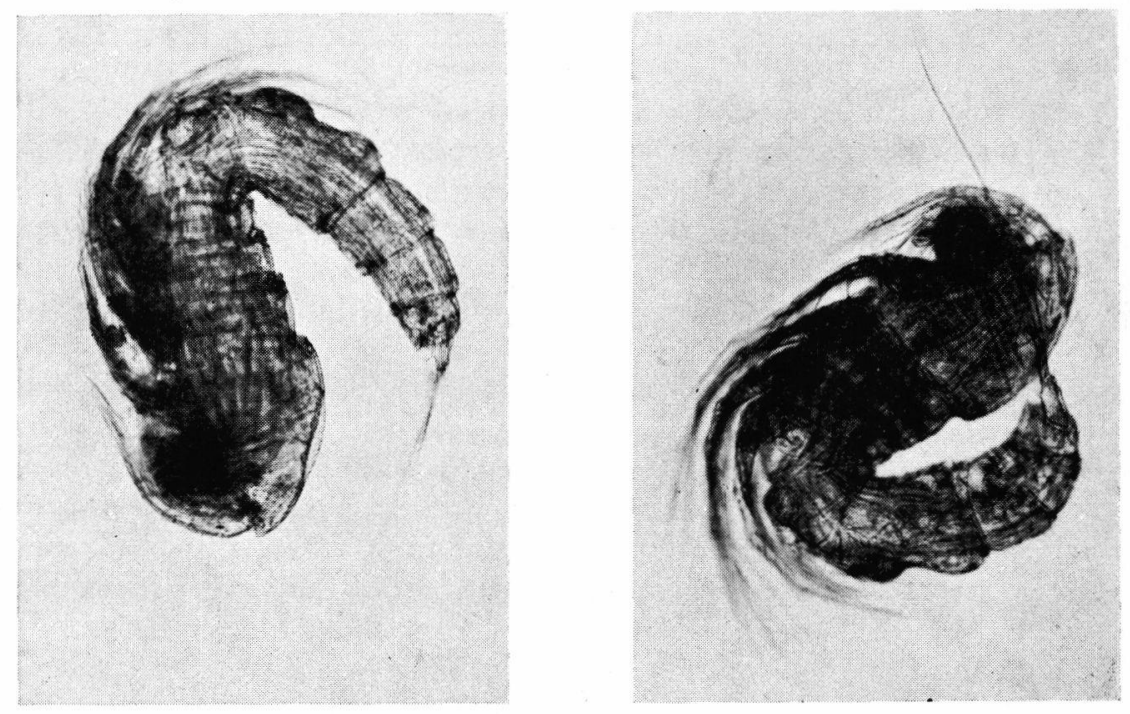

Fig. 6. - Canthocamptus staphylinus : individus présentant une flexion dorsale du post-abdomen.

\section{DISCUSSION}

L'examen des cycles biologiques montre que la reproduction et la croissance des populations de Cladocères et de Copépodes étudiées s'effectuent dans l'intervalle d'environ quatre mois, période où les eaux sont dégagées des glaces. Le cycle vitak de la majorité des espèces se déroulant dans un laps de temps assez bref du fait des conditions extrêmes du milieu, est relativement simple avec, dans la plupart des cas, une seule génération annuelle. Parmi les facteurs de l'environnement influençant le comportement des espèces, il semble que le facteur thermique soit primordial.

Chez les Cladocères il règle nettement la reprise d'activité des espèces comme le montre le tableau suivant : 
Date d'apparition des premières $q$ ovigères

dégel : 13 juillet dégel : 30 juin

Alona affinis

(pop. litt.)

Alona affinis

(pop. litt. prof.)

Acroperus harpae

(pop. litt. prof.)

Eurycercus lamellatus

(pop. litt. prof.)
26 août

19 août

19 août

19 août
21 juillet

28 juillet

28 juillet

28 juillet

Le décalage de deux semaines enregistré entre les années 1970 et 1971 dans la période de dégel se retrouve dans les dates d'apparition des premières $q$ ovigères. Le même processus a été observé au niveau du phytoplancton (Capblancq 1972) et des populations de Chironomides (Laville 1971).

Le facteur thermique intervient également dans le déroulement du cycle. Si l'on compare le cycle vital des deux populations, littorale et de profondeur, d'Alona affinis (fig. 7), on constate que non seulement les premières femelles parthénogénétiques apparaissent plus précocement en zone littorale mais encore que la gamogenèse s'y produit également plus tôt. Cependant, les maxima de reproduction parthénogénétique et de reproduction sexuée ont lieu, en zone «littorale» comme en zone «littori-profonde» à la même époque, respectivement début septembre et fin septembre, période où les conditions thermiques sont semblables dans les deux zones. En ce qui concerne cette espèce, il semble donc qu'il se produise un phénomène d'ajustement et de rattrapage chez la population de profondeur, qui, à l'entrée de l'hiver, présente une structure analogue à celle des autres espèces avec formation de formes de résistance.

Effectivement à l'entrée de l'hiver, d'une manière générale, les espèces adoptent des processus particuliers leur permettant de résister aux conditions défavorables du milieu.

Ainsi la production d'œufs de durée constitue probablement chez les Cladocères la condition nécessaire pour assurer la survie des espèces, encore qu'il soit difficile, dans le cadre de cette étude, de rattacher ce phénomène aux facteurs particuliers de l'environnement ou à un mécanisme d'ordre génétique.

Les Copépodes peuvent entrer en phase de dormance. En effet, les Cyclopides paraissent aborder dès octobre une longue phase de repos hivernal, pendant laquelle ils subsistent à l'état de vie ralentie sous forme d'adultes ou de stades sub-adultes. De nombreux facteurs peuvent intervenir dans la mise en état de dormance chez 

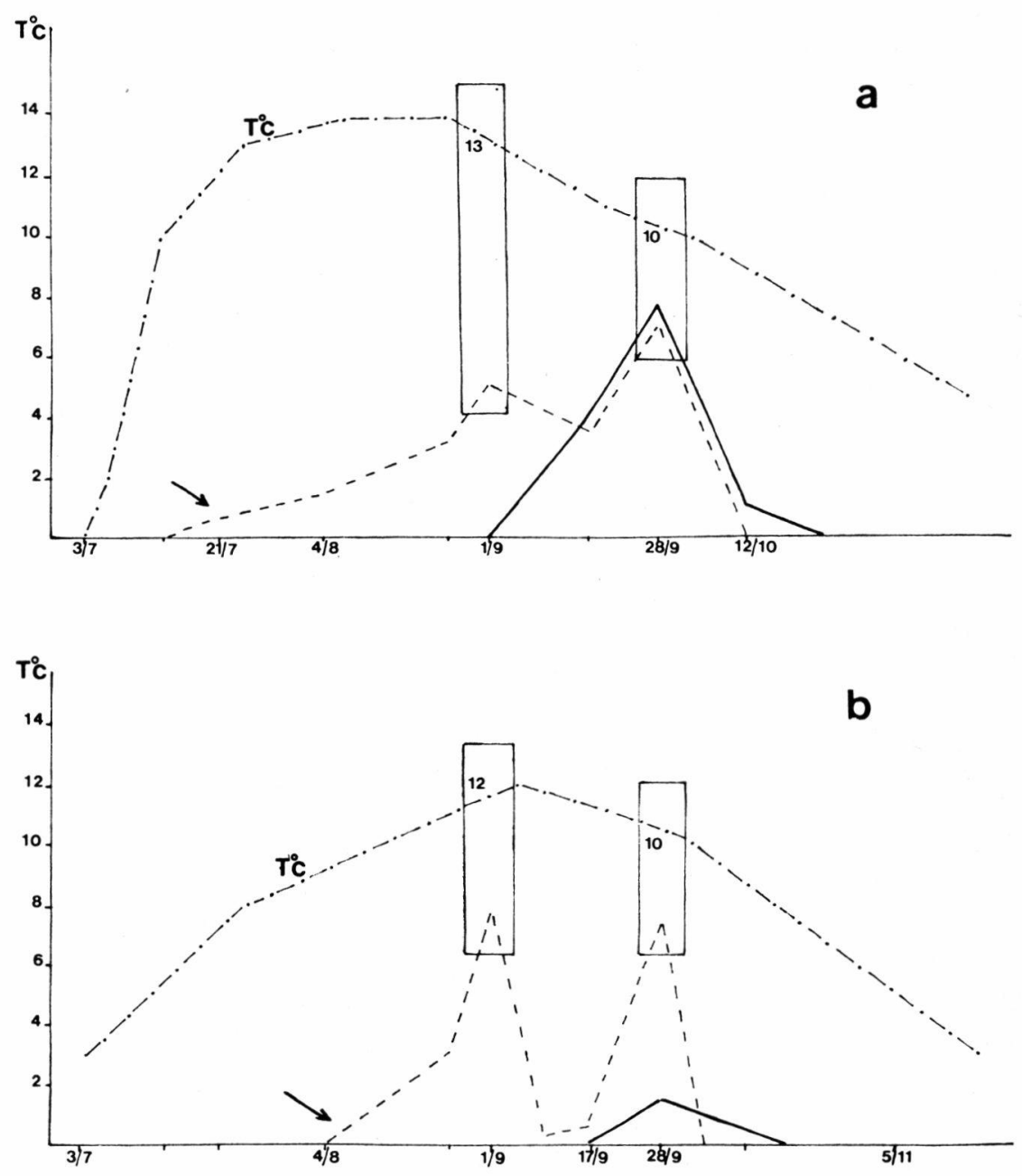

Fig. 7. - Evolution comparée des deux populations d'Alona affinis : a) population «littorale»; b) population de la zone «littori-profonde».

- . - . : : parthénogénétiques; : фo éphippiales.

les Copépodes, les espèces répondant spécifiquement aux différents facteurs.

Des expériences effectuées sur Eucyclops serrulatus (Auvray et Dussart 1966) ont montré un arrêt de la reproduction et une réduction du métabolisme à des températures inférieures à $5{ }^{\circ} \mathrm{C}$. Elgmork (1959) constate que Cyclops strenuus entre en diapause, dans le lac Bergstjern, sous la forme copépodite IV. Selon Wierz- 
bicka et Kedzierski (1964), un manque d'oxygène en profondeur provoquerait un état de dormance chez Cyclops bohater et $C$. vicinus vicinus. Einsle (1964) souligne l'importance de la photo-périodicité dans l'induction de la diapause chez les stades jeunes de Cyclops vicinus.

L'observation dans le lac de Port-Bielh (prélèvements du 2 juin et du 5 novembre 1971) d'individus présentant un faciès caractéristique de l'état léthargique : abdomen replié contre le céphalothorax (Dussart 1967), semble confirmer cette phase de dormance. Le fait en outre que les adultes of et $\&$ de septembre n'entrent pas en reproduction paraît indiquer une sorte de «mise en attente » de ces populations qui s'adapteraient ainsi aux conditions rigoureuses du milieu en période hivernale : basses températures, faibles teneurs en $\mathrm{O}_{2}$ (taux inférieur à $5 \mathrm{mg} / \mathrm{l}$ ).

Ce phénomène de vie ralentie est nettement caractérisé chez les Harpacticides par la formation de kystes. L'enkystement de Canthocamptus staphylinus a été signalé par de nombreux auteurs : Lauterborn et Wolf (1908), Kessler (1913), Berg (1938), Fryer et Smyly (1954), Smyly (1957). De ces différentes observations il ressort que le processus d'enkystement chez Canthocamptus staphylinus correspond à une élévation des températures. Le phénomène cesse dès que les individus sont soumis à une température de $9-10{ }^{\circ} \mathrm{C}$ (Kessler 1913 ).

Le comportement du Canthocamptus staphylinus peuplant le lac de Port-Bielh est différent. Outre les kystes d'été formés lorsque les températures sont maximales, l'espèce présente des kystes hivernaux alors que la température est de $4{ }^{\circ} \mathrm{C}$. Si donc l'on envisage l'influence du facteur thermique, il faut admettre que, au même titre qu'une élévation, une trop forte diminution des températures peut également entraîner chez cet Harpacticide une phase de dormance et d'enkystement.

\section{TRAVAUX CITES}

Auvray (C.) et Dussart (B.). 1966, - Rôle de quelques facteurs du milieu sur le développement post-embryonnaire de Cyclopides (Crustacés, Copépodes). I. Généralités, cas des Eucyclops. Bull. Soc. Zool. Fr., 91 (3) : 477-491.

Berg (K.). 1938. - Studies on the Bottom Animal of Esrom Lake. Mem. Acad. R. Sc. Lett. Danemark Sect. Sc., go série, 8 :1-255.

Capblance (J.). 1972. - Phytoplancton et productivité primaire de quelques lacs d'altitude dans les Pyrénées. Annls Limnol., 8 (3) : 231-321.

Capblancp (J.) et Laville (H.). 1968. - Étude morphométrique et physico-chimique de neuf lacs du massif de Néouvielle (Hautes-Pyrénées). Annls Limnol., 4 (2) : 275-324. 
Capblance (J.) et Laville (H.). 1972. - Étude de la productivité du lac de Port-Bielh (Pyrénées centrales). Proc. I.B.P. - U.N.E.S.C.O., Symposium on Productivity Problems of Freshwaters, KazimierzDolny, Poland. May 6-12, $1970: 73-88$.

Dussart (B.). 1967. - Les Copépodes des eaux continentales d'Europe occidentale. Tome I : Calanoides et Harpacticoides. Boubée \& Cle, Ed., 500 p.

Einsle (U.). 1964. - Die Gattung Cyclops s. str. im Bodensee. Arch. Hydrobiol., 60 (2) : 131-169.

ELGMORK (K.). 1959. - Seasonal occurence of Cyclops strenuus in relation to environment in small water bodies in Southern Norway. Folia Limnol. Scand., 11 : 196 p.

Fryer (G.) et Smyly (W. J. P.). 1954. - Some remarks on the resting stages of some freshwater Cyclopoid and Harpacticoid Copepods. Ann. Mag. Nat. Hist., 12 (7) : 65-72.

Gouxetre (P.). 1966. - Les Copépodes des lacs d'Estibère. D.E.S. Toulouse, 35 p.

Kessler (E.). 1913. - Über einige Harpacticiden des Riesengebirges. Zool. Anz., $\mathrm{n}^{\circ} 2: 72-75$.

Lauterborn (R.) et Wolf (E.). 1908. - Cystenbildung bei Canthocamptus microstaphylinus. Zool. Anz., $34:$ 130-136.

LAville (H.). 1971. -- Recherches sur les Chironomides (Diptera) lacustres du massif de Néouvielle (Hautes-Pyrénées): Systématique, Ecologie, Phénologie. Annls Limnol., 7 (2) : 173-332.

Rey (J.). 1968. - Ecologie des Cladocères du massif de Néouvielle (Hautes-Pyrénées). Annls Limnol., 4 (3) : 325 356.

Rey (J.) et Dupin (B.). 1973. - Écologie des Crustacés benthiques du lac de Port-Bielh (Pyrénées centrales): I. Répartition. Annls Limnol., 9 (2) : 121-134.

Smyly (W. J. P.). 1957. - Observations on the life-history of the Harpacticoid Copepod, Canthocamptus staphylinus (Jurine). Ann. Mag. Nat. Hist., $12^{\circ}$ sér., 10: 509-512.

Wierzbicka (M.) et Kedzierski (S.). 1964. - On the dormancy state of some species of Cyclopoida under experimental and natural conditions. Polsk. Arch. Hydrobiol., 12 (1) : 47-80. 